\title{
Functional Assessment In Vivo of the Mouse Homolog of the Human Ala-9-Ser NHE6 Variant
}

\author{
Qing Ouyang, , ${ }^{1,2,3}$ Lena Joesch-Cohen,, ${ }^{1,4}$ Sasmita Mishra, , ${ }^{1,2,3}$ Hasib A. Riaz, ${ }^{1,2,3}$ Michael Schmidt, ${ }^{1,2,3,4}$ \\ and (1)Eric M. Morrow ${ }^{1,2,3,4,5}$
}

https://doi.org/10.1523/ENEURO.0046-19.2019

\begin{abstract}
${ }^{1}$ Department of Molecular Biology, Cell Biology and Biochemistry, Brown University, Providence, RI 02912, ${ }^{2}$ Center for Translational Neuroscience, Robert J. and Nancy D. Carney Institute for Brain Science and Brown Institute for Translational Science, Brown University, Providence, RI 02912, ${ }^{3}$ Developmental Disorders Genetics Research Program, Emma Pendleton Bradley Hospital, East Providence, RI 02915, ${ }^{4}$ Hassenfeld Child Health Innovation Institute, Brown University, Providence, RI 02912, and ${ }^{5}$ Department of Psychiatry and Human Behavior, The Warren Alpert Medical School of Brown University, Providence, RI 02912
\end{abstract}

\begin{abstract}
Christianson syndrome (CS) is an X-linked neurogenetic disorder resulting from loss-of-function (LoF) mutations in SLC9A6, which encodes the endosomal $\mathrm{Na}^{+} / \mathrm{H}^{+}$exchanger 6 (NHE6). NHE6 regulates proton efflux from endosomes and, thus, participates in regulating cargo processing and trafficking. LoF mutations in NHE6 cause aberrant acidification of endosomes. While CS arises in males generally due to clear LoF mutations, other potentially hypomorphic variants have emerged, yet most of these variants have not been evaluated for functional effects, particularly in vivo. Here we characterize an SLC9A6 variant that has been previously reported in patients, yet now also appears in exome datasets of largely control individuals-c.25G>T, p.A9S. By heterologous expression in cell lines, we show that human NHE6A9S is expressed and localizes in a manner comparable to control NHE6. By genome editing, we generated the equivalent NHE6 mutation in mouse-p.A11S-and determined that male NHE6A11S mice have normal brain size at 6 months of age and do not show cerebellar degeneration or defective neuronal arborization. Neurons from male NHE6A11S mice also did not demonstrate an abnormality in intraendosomal pH compared with controls. These findings are in contrast to findings in NHE6-null mice previously reported and indicate that the NHE6A11S variant functions at a level equivalent to control NHE6 for many of the assays performed. These data stand in support of the population genetic data, which are also evaluated here, indicating that the A9S variant is unlikely to confer disease susceptibility with high penetrance.
\end{abstract}

Key words: Christianson syndrome; endosomes; genetics; SLC9A6; variant of unknown significance

\section{Significance Statement}

Loss-of-function mutations in SLC9A6, encoding $\mathrm{Na}^{+} / \mathrm{H}^{+}$exchanger 6 (NHE6), cause Christianson syndrome. Also, missense variants of unknown consequences have emerged. Functional evaluation of these mutations contributes to an understanding of the medical relevance and cellular effects of these variants. We show that human NHE6A9S protein is synthesized and localizes normally. Mice genome edited to the equivalent variant-NHE6A11S - did not show a reduction in brain size or defects in neuronal arborization. Mutant NHE6A11S neurons also did not demonstrate a significant decrease in luminal $\mathrm{pH}$ of endosomes. These findings are in contrast to findings in NHE6-null mice previously reported. Combined with the population genetic data, our data indicate that the NHE6A9S variant is unlikely to confer disease susceptibility with high penetrance. 


\section{Introduction}

Christianson syndrome (CS) is an X-linked neurogenetic disorder first described in a South African family in 1999 (Christianson et al., 1999). The core clinical features of CS include moderate to severe intellectual disability, nonverbal status, epilepsy, truncal ataxia, postnatal microcephaly, and hyperkinetic behavior (Christianson et al., 1999; Pescosolido et al., 2014). Secondary clinical features include eye movement abnormalities, motor regression, cerebellar atrophy, low body weight, and a happy demeanor resembling Angelman syndrome (AS; Christianson et al., 1999; Gilfillan et al., 2008; Schroer et al., 2010; Mignot et al., 2013; Pescosolido et al., 2014). The genetic basis of CS is loss-of-function (LoF) mutations in the $\mathrm{X}$-linked gene SLC9A6, which encodes the endosomal $\mathrm{Na}^{+} / \mathrm{H}^{+}$exchanger 6 (NHE6; Gilfillan et al., 2008; Garbern et al., 2010; Takahashi et al., 2011; Tzschach et al., 2011; Mignot et al., 2013; Riess et al., 2013; Pescosolido et al., 2014; Zanni et al., 2014; Masurel-Paulet et al., 2016). Systematic sequencing of X-chromosome genes has indicated that CS is among the most common X-linked developmental brain disorders (Tarpey et al., 2009).

NHE6 belongs to the SLC9A gene family. NHE6 mainly localizes to early, recycling, and late endosomes, while also transiently localizing to the plasma membrane (Brett et al., 2002; Nakamura et al., 2005; Ohgaki et al., 2008, 2010; Ouyang et al., 2013). By moving $\mathrm{H}^{+}$out of the organellar lumen in exchange for $\mathrm{Na}^{+}$or $\mathrm{K}^{+}$, organellar NHEs such as NHE6 participate in regulating luminal $\mathrm{pH}$, which is an essential aspect of the processing and trafficking of intracellular cargo (Orlowski and Grinstein, 2004; Ohgaki et al., 2011; Kondapalli et al., 2014). In

Received February 6, 2019; accepted October 30, 2019; First published November 1, 2019.

The authors declare no competing financial interests.

Author contributions: Q.O., L.J.-C., and E.M.M. designed research; Q.O., L.J.-C., S.M., H.A.R., M.S., and E.M.M. performed research; Q.O., L.J.-C., S.M., M.S., and E.M.M. analyzed data; Q.O., L.J.-C., and E.M.M. wrote the paper.

This research was supported in part by National Institutes of Health/National Institute of Mental Health Grants R01-MH-102418, R01-MH-105442, and R21$\mathrm{MH}-115392$; a General Research Grant from the Angelman Syndrome Foundation; and the Hassenfeld Child Health Innovation Institute at Brown University (E.M.M.); and a Hassenfeld Summer Scholar Award (L.J.-C.). The Mouse Transgenic and Gene Targeting Facility at Brown University is supported by National Institutes of Health/National Institute of General Medical Sciences Grant P30-GM1-03410. The Opera Phenix High Content Screening System in the Center to Advance Predictive Biology at Brown University is supported by National Institutes of Health/National Institute of Environmental Health Sciences Grant U01-ES-028184, National Science Foundation EPSCoR Award 1655221, and a generous gift by Donna McGraw Weiss (1989) and Jason Weiss.

Acknowledgments: We thank Geoffrey Williams, at the Brown University Leduc Bioimaging Facility, and Dr. Judy S. Liu for advice regarding microscopy. We also thank the Exome Aggregation Consortium and the groups that provided exome variant data for comparison. A full list of contributing groups can be found at http://exac.broadinstitute.org/about.

Correspondence should be addressed to Eric $M$. Morrow at Eric_morrow@brown.edu.

https://doi.org/10.1523/ENEURO.0046-19.2019

Copyright (C) 2019 Ouyang et al.

This is an open-access article distributed under the terms of the Creative Commons Attribution 4.0 International license, which permits unrestricted use, distribution and reproduction in any medium provided that the original work is properly attributed. neurons, NHE6 is found in growing axons and dendrites and at branch points. It also colocalizes with presynaptic and postsynaptic markers (Deane et al., 2013; Ouyang et al., 2013), indicating that NHE6 is involved in many cellular processes critical to normal nervous system development and function.

More than 30 distinct mutations in SLC9A6 have been identified in CS patients (Gilfillan et al., 2008; Garbern et al., 2010; Schroer et al., 2010; Takahashi et al., 2011; Mignot et al., 2013; Schuurs-Hoeijmakers et al., 2013; Bosemani et al., 2014; Pescosolido et al., 2014; Zanni et al., 2014; Masurel-Paulet et al., 2016; Trump et al., 2016; Padmanabha et al., 2017; Kerner-Rossi et al., 2018; Weitensteiner et al., 2018). Most of the mutations in SLC9A6 are nonsense mutations thought to result in truncation of the NHE6 protein or destruction of NHE6 mRNA by nonsense-mediated decay; thereby, the majority of mutations are LoF mutations (Kondapalli et al., 2014; Pescosolido et al., 2014). As supported by mouse studies, the loss of NHE6 function results in overacidification of the endosomal compartment, attenuated TrkB signaling, decreased neuronal arborization and circuit strength (Ouyang et al., 2013), and, potentially, disruption of neurotransmitter receptor trafficking (Deane et al., 2013).

Other types of mutations and variants identified in SLC9A6 include in-frame deletions, missense mutations, and splicing mutations (Fichou et al., 2009; Riess et al., 2013; Pescosolido et al., 2014; Zanni et al., 2014; llie et al., 2016; Masurel-Paulet et al., 2016; Padmanabha et al., 2017; Weitensteiner et al., 2018). A limited number of these mutations have been assessed in vitro (e.g., expression in heterologous cell lines), with results indicating that the NHE6-mutant proteins are unstable and do not undergo appropriate protein maturation and that their expression leads to defects in the functioning and survival of cells (Gilfillan et al., 2008; Roxrud et al., 2009; llie et al., 2014, 2016, 2019). Molecular and cellular characterization of SLC9A6 variants such as missense variants will contribute to our understanding of their medical significance, as well as broaden our understanding of the function of NHE6.

The purpose of the study presented here was to evaluate a missense mutation of SLC9A6 reported by Fichou et al. (2009) wherein a c.25G $>$ T mutation in SLC9A6 exon 1 results in an alanine-to-serine substitution at position 9 of NHE6 (p.A9S). This mutation was identified in a cohort of male patients with AS-like features. Subsequently, however, the NHE6A9S variant has been found in large, putative control exome sequencing databases, calling into question the medical significance and disease-causing role of this variant. We provide results here based in expression of wild-type and mutant NHE6 in cultured cells and analysis of a mouse model with an equivalent mutation (p.A11S) indicating that the alanine-to-serine mutant NHE6 performs in a manner largely similar to wild-type NHE6 with respect to the tested functional measures. Our data, combined with the human population genetic data as evaluated here, provide support for the interpretation that this human gene variant is unlikely to confer susceptibility to disease with high pen- 
Table 1: Values for the A9S variant of SLC9A6 reported in the ExAC database

\begin{tabular}{ll}
\hline AN_female & 13,626 \\
AN_male & 1232 \\
AC_female & 92 \\
AC_male & 22 \\
AC_het & 92 \\
$q$ & 0.00767 \\
\hline
\end{tabular}

AC, Total number of chromosomes with variant allele; AN, total number of chromosomes able to be called regardless of whether reference or variant allele; $q$, frequency of variant allele.

etrance. As such, these results may assist in interpretation of genetic diagnostic information.

\section{Materials and Methods}

\section{Analysis of the population frequency and sex skew of the A9S variant in SLC9A6}

The total number of male (AN_male) and female (AN_female) chromosomes covered at the location of the A9S variant in SLC9A6, along with the number of mutant female alleles (AC_female), mutant male alleles (AC_male), and heterozygotes (AC_het), was extracted from the Exome Aggregation Consortium (ExAC) database (http://exac.broadinstitute.org/; Lek et al., 2016; Table 1). Based on the information in Table 1, the observed number of individuals with each possible genotype for this locus was calculated using the following equations:

$M$ (total number of male samples covered at this location) = AN_male,

F (total number of female samples covered at this location) $=\mathrm{AN} \_$female $/ 2$,

$$
\begin{gathered}
X(\text { mut }) / Y=A C \_ \text {male, } \\
X(\text { wt }) / Y=M-A C \_m a l e, \\
X(\text { mut }) / X(w t)=A C \_h e t, \\
X(\text { mut }) / X(\text { mut })=(\text { AC_female }- \text { AC_het }) / 2,
\end{gathered}
$$$$
\text { and }
$$

$X(w t) / X(w t)=F-(X(m u t) / X($ mut $)+X($ mut $) / X(w t))$.

The results of the above calculations are shown in Table 2 under the "Observed" column.

To calculate the expected number of individuals of each genotype, Hardy-Weinberg equilibrium was assumed, with $q$ (the frequency of the mutant allele) given by ExAC, as reported in Table 1. This frequency value was used in conjunction with the $F$ and $M$ values defined above and the following equations:

$$
\begin{gathered}
\mathrm{p} \text { (frequency of wild-type allele) }=1-\mathrm{q}, \\
\mathrm{X}(\text { wt }) / Y=M * \mathrm{p}, \\
X(\text { mut }) / Y=M * \mathrm{q},
\end{gathered}
$$

Table 2: Observed and expected values for different genotypes of the A9S variant of SLC9A6

\begin{tabular}{lcc}
\hline \multicolumn{1}{c}{ Genotype } & Observed & Expected \\
$X(\mathrm{wt}) / X(\mathrm{wt})=$ homozygous wild-type & 6721 & 6709 \\
$\mathrm{female}$ & & \\
$X(\mathrm{wt}) / X(\mathrm{mut})=$ heterozygous female & 92 & 104 \\
$X(\mathrm{mut}) / X(\mathrm{mut})=$ homozygous mutant & 0 & 0 \\
$\mathrm{female}$ & & \\
$X(\mathrm{wt}) / Y=$ hemizygous wild-type male & 1210 & 1223 \\
$X(\mathrm{mut}) / Y=$ hemizygous mutant male & 22 & 9 \\
\hline
\end{tabular}

mut, Mutant; wt, wild-type.

$$
\begin{gathered}
\mathrm{X}(\mathrm{mut}) / \mathrm{X}(\mathrm{mut})=\mathrm{F} *\left(\mathrm{q}^{\wedge} 2\right), \\
\mathrm{X}(\mathrm{mut}) / \mathrm{X}(\mathrm{wt})=\mathrm{F} * 2 * \mathrm{p} * \mathrm{q}, \\
\text { and } \\
\mathrm{X}(\mathrm{wt}) / \mathrm{X}(\mathrm{wt})=\mathrm{F} *\left(\mathrm{p}^{\wedge} 2\right) .
\end{gathered}
$$

The results of the above calculations are shown in Table 2 under the "Expected" column.

\section{Cell line and mouse lines}

For experiments involving use of cultured cells, the HeLa cell line was used (CCL-2, ATCC; RRID: CVCL_0030). For studies in the mouse, male mice from the following two Nhe6 mouse lines were used: a mouse model of the human A9S variant (A11S in mouse) of NHE6; and a new NHE6-null mouse model due to a 4 bp CAAG deletion in exon 15 that causes a frameshift and subsequent generation of a premature stop codon (ChrX: g.56658353-56658356, c.1825-1828, GRCm38; NCBI Reference Sequence: NC_000086; Ensembl Transcript: ENSMUST00000077741.11). These mouse lines were generated using CRISPR/Cas9-mediated genome editing (Mouse Transgenic and Gene Targeting Facility of Brown University) and are described for the first time herein. Both mouse lines are on the C57BL/6N mouse background. The targeting of constructs and the presence of mutations were confirmed by PCR genotyping and Sanger sequencing. Western blotting was used to confirm protein expression or lack thereof (see Fig. 2). All experiments involving live animals were conducted in accordance with the U.S. National Institutes of Health Guide for the Care and Use of Laboratory Animals (National Research Council of the National Academies, 2011) under a protocol approved by the Brown University Animal Care and Use Committee.

\section{DNA constructs}

The human NHE6 gene (GenBank: NM_006359) was cloned into a pReceiver-M07 mammalian expression vector to generate a C-terminal HA-tagged pNHE6-HA construct. The pNHE6-HA construct was then used, together with the QuickChange site-directed mutagenesis kit (Stratagene), to generate a construct encoding for an HAtagged version of the A9S variant of human NHE6 (NHE6A9S$\mathrm{HA}$ ). Mutagenesis was performed according to the manufacturer protocol and using the following overlapping primers: 
(5'-GCGGAGGGGTGACCGCCGCCAGC-3') and (5'GCTGGCGGCGGTCACCCCTCCGC-3').

\section{PCR genotyping}

To confirm the $G>T$ mutation targeting strategy in the NHE6A11S-mutant mouse model, PCR genotyping was performed using mouse tail lysates and the following primers: 141_Slc9a6_ex1F1 (5'-CTGTAGGTGGGTAGACAAGCC-3') and 147_SIc9a6_ex1R4 (5'-TTCTCGGACACGATCTCCTC-3'). Target bands of 402 bp were gel purified, followed by Sanger sequencing to determine genotype. The 4 bp deletion in the NHE6-null mouse model was confirmed by PCR genotyping using the following primers: 522_Slc9a6_ex15uF3 (5'CAGTAGAGCACTTGTAGAGCAC-3') and 555_SIc9a6_ex15dR5 (5'-CAGAAGCCTAACCCATAGAAC$\left.3^{\prime}\right)$. Target bands of 406 bp were gel purified, followed by Sanger sequencing to determine genotype.

\section{Western blotting}

Mouse whole-brain lysates or HeLa cell lysates were prepared and subsequently heated at $70^{\circ} \mathrm{C}$ for $10 \mathrm{~min}$ in $4 \times$ NuPage LDS sample buffer (catalog \#NP0007, Invitrogen) with $10 \times$ NuPage sample reducing agent (catalog \#NP0009, Invitrogen). Samples were then subjected to SDS-PAGE in a $4-12 \%$ polyacrylamide gel and transferred to nitrocellulose membranes according to standard procedures. Western blotting was performed as follows. The membrane was blocked for $1 \mathrm{~h}$ at room temperature using Odyssey TBS blocking buffer (catalog \#927-50000, $\mathrm{LI}-\mathrm{COR}$ ), incubated overnight at $4^{\circ} \mathrm{C}$ in primary antibody diluted in TBS blocking buffer, rinsed $3 \times 10$ min with TBS-Tween, incubated for $1 \mathrm{~h}$ at room temperature in secondary antibody diluted in TBS blocking buffer, rinsed $3 \times 10$ min with TBS-Tween, and scanned with an Odyssey CLx Infrared Imaging System (LI-COR; RRID: SCR_014579). The primary antibodies used were rabbit anti-NHE6 (048; 1:1000 working dilution; Ouyang et al., 2013), mouse anti- $\alpha$-tubulin (1:4000 working dilution; catalog \#T6074, Sigma-Aldrich; RRID:AB_477582), and rabbit anti-HA (1:1000 working dilution; catalog \#3724s, Cell Signaling Technology; RRID:AB_1549585). The secondary antibodies used were goat anti-rabbit IRDye680 (1: 20,000 working dilution; catalog \#925-68071, LI-COR; RRID:AB_2721181) and goat anti-mouse IRDye800 (1: 20,000 working dilution; catalog \#925-32210, LI-COR; RRID:AB_2687825).

\section{Immunocytochemistry}

For cultured HeLa cells, cells were first rinsed twice with $1 \times$ PBS. Cells were then fixed in $4 \%(w / v)$ paraformaldehyde (PFA) for $15 \mathrm{~min}$ and permeabilized with $0.25 \%(\mathrm{w} / \mathrm{v})$ Triton X-100 in $1 \times$ PBS for $15 \mathrm{~min}$ at room temperature. Cells were then blocked with $10 \%$ normal goat serum in $1 \times$ PBS $+0.1 \%$ Triton X-100 (PBST) for $1 \mathrm{~h}$ at room temperature followed by incubation overnight at $4^{\circ} \mathrm{C}$ with primary antibodies diluted in PBST containing $2 \%$ normal goat serum. The primary antibodies used were rabbit anti-NHE6 (048; Ouyang et al., 2013) and mouse anti-HA (catalog \#11583816001, Roche; RRID:AB_514505). After rinsing $3 \times 5 \mathrm{~min}$ in PBST, cells were incubated for $1 \mathrm{~h}$ at room temperature with secondary antibodies diluted in
PBST containing $2 \%$ normal goat serum. The secondary antibodies used were goat anti-rabbit Alexa Fluor 488 (1:800 working dilution; catalog \#A-27034, Thermo Fisher Scientific; RRID:AB_2536097) and goat anti-mouse Alexa Fluor 594 (1:800 working dilution; catalog \#A-11032, Thermo Fisher Scientific; RRID:AB_2534091). Additionally, nuclei were counterstained with DAPI (1:1000 working dilution; Thermo Fisher Scientific) at the same time as incubation with secondary antibody. Cells were again rinsed $3 \times 5 \mathrm{~min}$ in PBST and then mounted in Fluoromount-G (SouthernBiotech).

For hippocampal cultures, wild-type and NHE6A11Smutant neurons were first starved with Earle's balanced salt solution (EBSS) for $30 \mathrm{~min}$, and then cells were incubated with $25 \mu \mathrm{g} / \mathrm{ml}$ Alexa Fluor 568-conjugated transferrin (catalog \#T23365, Thermo Fisher Scientific) for $10 \mathrm{~min}$. After washing three times with $1 \times$ PBS, cells were fixed with 4\% PFA and immunostained using rabbit anti-NHE6 as the primary antibody (048; Ouyang et al., 2013) and goat anti-rabbit Alexa Fluor 488 as the secondary antibody (1:800 working dilution; catalog \#A-27034, Thermo Fisher Scientific; RRID:AB_2536097). Nuclei were counterstained with DAPI (1:1000 working dilution; Thermo Fisher Scientific).

Confocal microscopy images were collected using a Zeiss LSM 710 confocal microscope. Structured illumination microscopy (SIM) images were collected using a DeltaVision OMX SR microscope. Z-series images were collected using a $60 \times$ oil objective (refractive index immersion oil 1.516) and structured illumination (SI) light path under sequential acquisition mode. Z-series images were processed by performing OMX SI reconstruction, alignment, and maximal projection sequentially using softWoRx software. Images were analyzed using ImageJ software (NIH).

\section{Tissue preparation}

Mice were anesthetized with Beuthanasia-D and transcardially perfused with phosphate-buffered 4\% PFA. Brains were removed from skulls, stored in 4\% PFA overnight at $4^{\circ} \mathrm{C}$, sequentially immerged in $10 \%, 20 \%$, and $30 \%$ sucrose solutions at $4^{\circ} \mathrm{C}$ for $1 \mathrm{~d}$ per solution of a specific percentage of sucrose, embedded in optimal cutting temperature compound, and stored at $-80^{\circ} \mathrm{C}$.

\section{Brain size measurement}

Matched male littermate pairs collected at 6 months of age were transcardially perfused with $4 \%$ PFA and imaged using a Leica MZ16F dissecting microscope. The areas of the cortical hemispheres, midbrain, and cerebellum were measured using ImageJ software $(\mathrm{NIH})$.

\section{Histology and immunohistochemistry}

For cerebellar degeneration analysis, $30 \mu \mathrm{m}$ sagittal sections were collected using a Leica Cryostat. To perform immunohistochemistry, sections were first prewetted with $1 \times$ PBS for $15 \mathrm{~min}$. Sections were then postfixed with $4 \%$ PFA for $15 \mathrm{~min}$, permeabilized with $0.25 \%(\mathrm{w} / \mathrm{v})$ Triton X-100 in $1 \times$ PBS for 15 min, blocked with $10 \%$ normal goat serum in PBST for $2 \mathrm{~h}$ at room temperature, and incubated overnight at $4{ }^{\circ} \mathrm{C}$ with mouse anti-calbindin 
antibody diluted in PBST containing $2 \%$ normal goat serum (1:1000 working dilution; Swant 300, Swant; RRID: $\left.A B \_10000347\right)$. After washing the sections extensively with PBST, they were incubated with Alexa Fluor 568conjugated goat anti-mouse secondary antibody diluted in PBST containing 2\% normal goat serum (1:800 working dilution; catalog \#A-11004, Thermo Fisher Scientific; RRID:AB_2534072) for $2 \mathrm{~h}$ at room temperature. The sections were then washed three times with PBST and mounted using Fluoromount-G (SouthernBiotech).

\section{Endosomal pH, transferrin distribution, and endosome size analyses in mouse hippocampal neurons using the Opera Phenix High-Content Screening System}

Isolated hippocampal neurons were seeded at a density of 28,000 cells/well in CellCarrier-96 Ultra Microplates (clear bottom black; PerkinElmer). For each pup, three to four wells of cells were seeded and analyzed, which served as a means of experimental replication. At $5 \mathrm{~d}$ in vitro (DIV), cells were first starved in EBSS at $37^{\circ} \mathrm{C}$ for 30 min. EBSS was then replaced with regular Neurobasal-A media containing $33 \mu \mathrm{g} / \mathrm{ml}$ fluorescein isothiocyanate (FITC)-conjugated transferrin (catalog \#T2871, Thermo Fisher Scientific), which is $\mathrm{pH}$ sensitive, and $33 \mu \mathrm{g} / \mathrm{ml}$ Alexa Fluor 546-conjugated transferrin (catalog \#T23364, Thermo Fisher Scientific), which is $\mathrm{pH}$ insensitive. Nuclei were simultaneously counterstained with DAPI to facilitate data analysis. After a 10 min incubation, the cells were washed twice with warm PBS, placed in media lacking phenol red, and imaged live under 60× water objective using an Opera Phenix High-Content Screening System (PerkinElmer). Twenty images were taken per well. To generate the standard curve for use in determining endosomal $\mathrm{pH}$, similar procedures were followed; however, neurons were imaged in standard buffer solutions containing the following: $125 \mathrm{~mm} \mathrm{KCl}, 25 \mathrm{~mm} \mathrm{NaCl}, 10 \mu \mathrm{M}$ monensin, and either $25 \mathrm{~mm}$ HEPES, for a standard $\mathrm{pH}$ value of 7.0 , or $25 \mathrm{~mm}$ MES, for standard $\mathrm{pH}$ values of 6.5 , 6.0 , and 5.5 , and adjusted to a final $\mathrm{pH}$ using $1 \mathrm{~N} \mathrm{NaOH}$ or $1 \mathrm{~N} \mathrm{HCl}$.

Confocal images were analyzed using Harmony software (PerkinElmer). The DAPI channel was used to identify nuclei and the somal region using the Find Nuclei and Find Cytoplasm building blocks. Detected nuclei were used to count cells in the assays, and a minimum nuclear area threshold criterion was applied to select for live cells. Transferrin-labeled endosomes in soma and neurites were identified using the Find Spots building block. To specify which kind of spots should be detected, the following parameters were adjusted: (1) specify region (soma or neurites) for the spots; and (2) specify the filter criteria to select spots by their fluorescence intensity and area. A spot area cutoff of 15-90 square pixels was chosen to represent endosomes, and the fluorescence intensity of each fluorophore was measured within endosomes. Also, data regarding the number of transferrinlabeled endosomes in neurites and soma and the live cell number were collected. To determine endosomal $\mathrm{pH}$, the ratio of FITC fluorescence intensity to Alexa Fluor 546 fluorescence intensity was calculated. For the standard curve, the ratio was plotted against $\mathrm{pH}$ values; for experimental measures, the ratio was extrapolated to the standard curve. To assess transferrin distribution, endosome number and localization (soma or neurite) were determined in live cells based on the Alexa Fluor 546conjugated transferrin signal. To compare the sizes of endosomes, area values for the spots analyzed were exported and square pixels were converted to square micrometers.

\section{Cerebellum image quantification}

The "Sagittal Atlas" from the Allen Mouse Brain Atlas was used to determine sagittal sections of the cerebellum to analyze (Allen Institute for Brain Science, 2004). Sections selected for mid-sagittal analyses most closely resembled Position 195 and Position 201 from the Sagittal Atlas (http://mouse.brain-map.org/experiment/thumbnails/100042147?image_type=atlas). Images of the cerebellum were acquired as $z$-stacks comprising $18 \times 1.63$ $\mu \mathrm{m}$ slices using an Olympus FV3000 confocal microscope equipped with a $20 \times$ objective. Individual images were stitched into a whole image. Purkinje cell (PC) body density was calculated as the number of PC bodies divided by the length of the PC layer measured. All images were analyzed using Image software $(\mathrm{NIH})$.

\section{Continuous live-cell imaging and neurite outgrowth analysis}

Continuous live-cell imaging was performed using an IncuCyte S3 Live-Cell Analysis System (Essen BioScience). For analysis of neurite outgrowth, 20,000 cells isolated from postnatal day 1 (P1) mouse hippocampi were seeded into 96-well plates. For each pup, four wells of cells were seeded and analyzed, which served as a means of experimental replication. Plates were placed into the IncuCyte S3 Live-Cell Analysis System at 1 DIV, and neurite outgrowth was followed by automated continuous live-cell imaging. Four phase-contrast images per well were imaged at intervals of $6 \mathrm{~h}$ with a $10 \times$ objective lens for 15 DIV in total. For neurite outgrowth analysis, recorded images were analyzed using the automated NeuroTrack image acquisition module of the IncuCyte Zoom software. The NeuroTrack algorithm automatically defines cell bodies and neurite extensions (see Fig. $5 \mathrm{C}$ ) to calculate and quantify the collective neurite length and neurite branch points per cell body cluster. Neurite length and neurite branch points were normalized to values for these parameters at time $0 \mathrm{~h}$; data are presented as fold $x$ values at time $0 \mathrm{~h}($ time $0 \mathrm{~h}=1)$.

\section{Statistical analysis}

A summary of statistical analyses relating to this study is provided in Table 3. Two-tailed Student's $t$ tests were performed for all group comparisons. For neurite length and neurite branch point measures (see Fig. 5A,B), two-way ANOVA followed by Tukey's multiple comparison tests were performed using GraphPad Prism 7 to compare the means for each genotype at each time point to one another. Data are presented as the mean \pm SEM. 
Table 3: Summary of statistical analyses

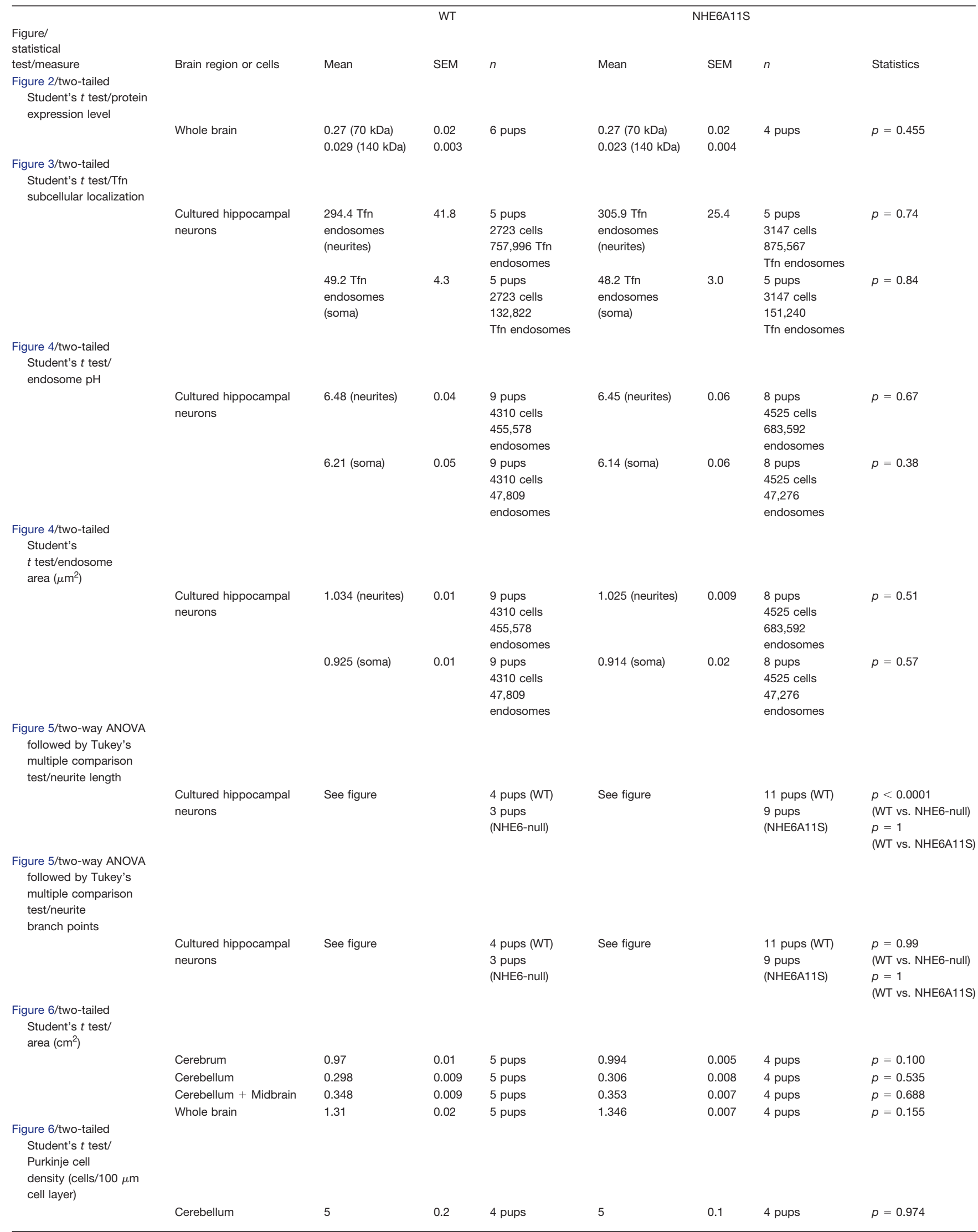

Tfn, Transferrin; WT, wild-type. 
A

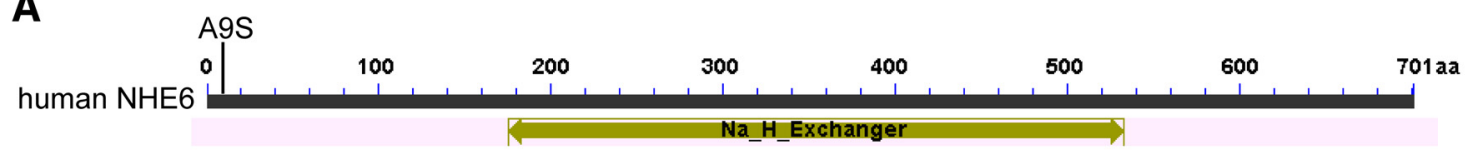

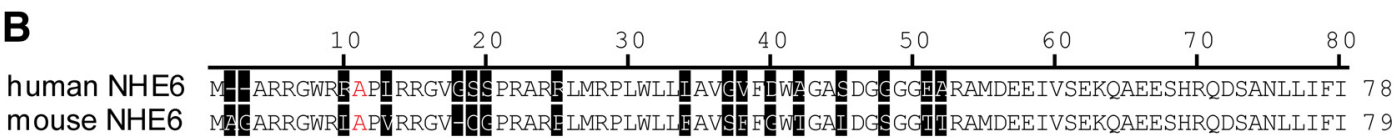

C

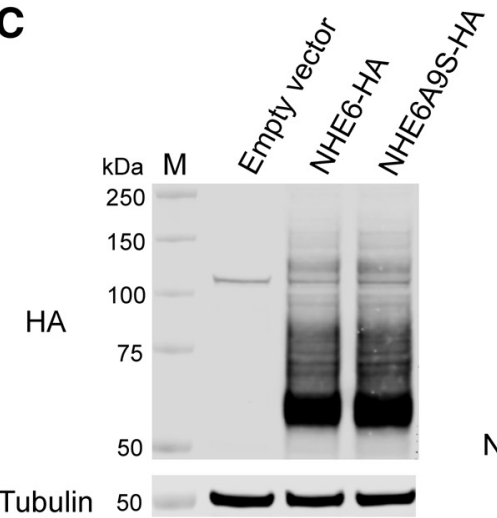

D

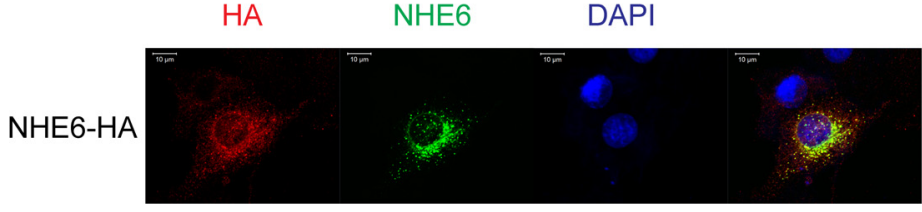

NHE6A9S-HA

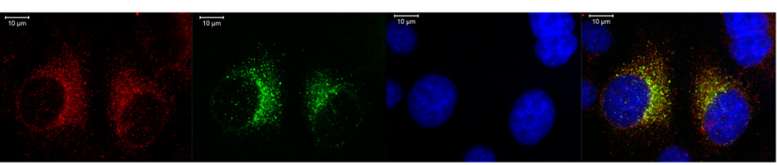

Figure 1. Evaluation of the NHE6A9S mutant in cultured cells. $\boldsymbol{A}$, Bar diagram of human NHE6. The predicted region of the conserved $\mathrm{Na}^{+} / \mathrm{H}^{+}$exchanger domain (gold arrow) and the alanine-to-serine mutation at position 9 (A9S) are shown. $\boldsymbol{B}$, Alignment of amino acid sequences of human NHE6 and mouse NHE6 from the predicted $\mathrm{N}$ terminus through to the end of the second transmembrane domain. The alanine $(\mathrm{A})$ at position 11 in mouse NHE6 corresponds to the alanine (A) at position 9 in human NHE6 (both in red font). Amino acids not conserved between the sequences or shifts in a sequence for optimal alignment are shaded. $\boldsymbol{C}$, Western blot of lysates from HeLa cells expressing HA-tagged human forms of wild-type NHE6 (NHE6-HA) or the NHE6A9S mutant (NHE6A9S-HA). Cells transfected with vector alone were used as a control. Lysates were probed with an anti-HA antibody to detect exogenously expressed NHE6 protein and an anti-tubulin antibody to detect tubulin, as a loading control. D, Confocal microscopy images of HeLa cells expressing NHE6-HA or NHE6A9S-HA and immunostained for HA (red) and NHE6 (green). Nuclei were counterstained using DAPI (blue). Scale bars, $10 \mu m$.

\section{Results}

\section{Population frequency analysis of the A9S variant in SLC9A6}

Fichou et al. (2009) identified a missense mutation (c.25G > T, p.A9S) in SLC9A6 in a cohort of male patients from white or North-African origin with AS-like features (1 of 59). This mutation is localized in SLC9A6 exon 1 and substitutes a polar amino acid, serine, for a nonpolar amino acid, alanine, at position 9 of the protein (Fig. 1A). The corresponding alanine in mouse NHE6 is at position 11 (Fig. 1B). It was proposed that alanine at position 9 (NP_006350.1) is highly conserved in several vertebrates, indicating that nonconservative mutation of this amino acid may alter the function of NHE6 (Fichou et al., 2009). To investigate whether the SLC9A6 c.25G $>$ T variant could be found in the general population, Fichou et al. (2009) screened 400 unaffected male individuals and identified 1 subject (of Egyptian origin) containing the variant. Combining the affected and unaffected male populations, the SLC9A6 c.25G $>$ T variant was suggested to be a rare polymorphism with an estimated frequency of $0.4 \%$ (2 of 459 individuals; Fichou et al., 2009).

The purpose of the study presented here was to investigate a larger control population in an effort to establish a better understanding of the genetic consequence of the c.25G $>$ T variant of SLC9A6. Using data contained in the ExAC database (http://exac.broadinstitute.org/; Lek et al., 2016), 14,858 SLC9A6 alleles were screened, and an allele count of 114 was determined with respect to the c.25G $>T$ variant, resulting in an estimated frequency of $0.767 \%$ (114 of 14,858 alleles; Table 1). The sex skew of the NHE6A9S variant was then calculated based on the data reported in the ExAC database (Table 2). Using the values presented in Table 1, together with the equations shown in Materials and Methods, the observed and expected numbers of individuals with each possible genotype for the c.25 locus of SLC9A6 were calculated. The results of these calculations are shown in Table 2. The results indicate that a greater number of males hemizygous for the NHE6A9S variant are observed than expected, whereas fewer females heterozygous for this variant are observed than expected. This suggests a lack of selective pressure against males hemizygous for the NHE6A9S variant. The predicted functional consequence of the NHE6A9S mutation was evaluated using PolyPhen-2 and SIFT. In using Polyphen-2, the NHE6A9S mutation is predicted to be benign. In using SIFT, however, this mutation is predicted to affect NHE6 function, although the number of sequences available for comparison was quite limited in using this program.

\section{In vitro functional evaluation of the NHE6A9S mutation}

To evaluate the effects of the NHE6A9S mutation on the function of NHE6 in an in vitro cellular model, the $\mathrm{G}>\mathrm{T}$ substitution was introduced into the cDNA of wild-type human SLC9A6 in a mammalian expression vector allow- 
ing for tagging of a protein at its $C$ terminus with $\mathrm{HA}$ (NHE6-HA for wild-type NHE6; NHE6A9S-HA for mutant NHE6). The constructs encoding for NHE6-HA and NHE6A9S-HA were then expressed in HeLa cells, and protein expression was evaluated by Western blotting. Wild-type NHE6 typically migrates as multiple bands when cell or tissue lysates are separated using SDSPAGE; these bands reflect the different glycosylated and oligomeric states of NHE6 (Ohgaki et al., 2008; llie et al., 2014). As presented in Figure $1 C$, HA-tagged NHE6A9S migrates as a smear of bands spanning from 60 to 150 $\mathrm{kDa}$, similar to wild-type HA-tagged NHE6, with dominant bands at $\sim 60,70$, and $125 \mathrm{kDa}$. These results suggest that NHE6A9S undergoes appropriate glycosylation and oligomerization. The expression levels of wild-type NHE6-HA and NHE6A9S-HA were also similar, suggesting similar levels of protein stability.

Previous studies have shown that NHE6 is detected as puncta localized throughout the cellular cytoplasm and also in the perinuclear region, likely reflecting its localization to various endosomal populations and trafficking through the Golgi apparatus for post-translational modification (Brett et al., 2002; Nakamura et al., 2005; Ohgaki et al., 2008, 2010; Ouyang et al., 2013). Results from immunocytochemistry and confocal microscopy-based imaging presented here indicate that NHE6A9S-HA localizes in a similar distribution pattern (Fig. 1D). Thus, the alanine-to-serine mutation does not appear to affect the subcellular localization of NHE6. Together, the results from these in vitro studies in HeLa cells support that the A9S mutation does not affect the maturation, stability, or intracellular localization of NHE6.

\section{Protein stability and subcellular localization of NHE6A11S in a mouse model}

To allow for analysis of the effects of the NHE6A9S variant on neuronal form and function and brain development, including in vivo analyses, a mouse model was generated using CRISPR/Cas9-mediated genome editing (Fig. 2A). In mouse NHE6, the corresponding alanine is in position 11 (Fig. 1B, amino acid sequence alignment). Thus, single guide RNAs (sgRNAs) were designed to introduce an A11S mutation into exon 1 of mouse S/c9a6 (Fig. 2A). PCR-based genotyping together with Sanger sequencing showed successful G-to-T genome editing in NHE6A11S-mutant mice (Fig. 2B), indicating that the desired missense mutation had been generated in mouse SIc9a6 using the CRISPR/Cas9 system. The assessment of NHE6 protein levels in mouse brain lysates by way of Western blotting supported that NHE6A11S-mutant protein, which is expressed from the endogenous S/c9a6 locus, is expressed to an equivalent level, is equally stable, and undergoes similar post-translational processing as wild-type NHE6 (Fig. 2C, quantification on right). In contrast, NHE6 is undetectable in Western blotting of brain lysate from mice of a newly generated Nhe6-null line due to a 4 bp deletion in exon 15 that results in a frameshift and subsequent generation of a premature stop codon (Fig. 2C; see Materials and Methods).
Next, the intracellular localization of NHE6A11S, compared with wild-type NHE6, was analyzed in hippocampal neurons isolated from mouse pups of the respective genotypes. As a marker for early endosomes, neurons were incubated with Alexa Fluor-conjugated transferrin for 10 $\min$. For fixed-cell analysis, neurons were then stained for NHE6 using immunocytochemistry and imaged using confocal microscopy (Fig. 3A) as well as SIM (Fig. 3B). As shown in Figure 3, NHE6A11S displayed a characteristic vesicular distribution that overlapped with transferrinpositive compartments, a distribution pattern similar to that of wild-type NHE6 in neurons (Ouyang et al., 2013). To check whether the expression of the NHE6A11S variant changes transferrin distribution in neurons with respect to soma versus neurites, we quantified the number of transferrin-positive endosomes in each region using a high-throughput confocal microscope-the Opera Phenix High-Content Screening System (see Materials and Methods). Our results showed that there is no significant difference in the distribution of transferrin-positive endosomes to neuronal soma and neurites in wild-type versus NHE6A11S-mutant neurons. Calculated numbers of transferrin-positive endosomes in each region for each genotype were as follows (numbers per cell are indicated): neurite transferrin-positive endosomes, $294.4 \pm 41.8$ (wild-type), $305.9 \pm 25.4$ (NHE6A11S); soma transferrinpositive endosomes, $49.2 \pm 4.3$ (wild-type), $48.2 \pm 3$ (NHE6A11S); $p=0.74$ for neurite transferrin-positive endosomes, $p=0.84$ for soma transferrin-positive endosomes. Data were obtained from five animals of each genotype from two independent litters (Table 3). Overall, these results support that, in mouse neurons, the A11S point mutation of NHE6 does not affect its stability, maturation, or intracellular localization.

\section{Intraendosomal pH in neurons from the NHE6A11S mouse model}

NHE6 has been identified to play an important role in the regulation of endosomal pH (Orlowski and Grinstein, 2004; Ohgaki et al., 2011; Kondapalli et al., 2014). As such, the effects of the expression of NHE6A11S on intraendosomal $\mathrm{pH}$ was tested, here, specifically in neurons. The $\mathrm{pH}$ of early endosomes in wild-type and NHE6A11S-mutant neurons was measured using a technique involving endocytosis of $\mathrm{pH}$-sensitive and $\mathrm{pH}$-insensitive fluorescent conjugates of transferrin and ratiometric imaging using the Opera Phenix High-Content Screening System and Harmony software (Fig. 4; see also Materials and Methods). For experimental measures, hippocampal neurons (wild-type and NHE6A11Smutant) were preloaded with FITC-conjugated ( $\mathrm{pH}$-sensitive) transferrin and Alexa Fluor 546-conjugated ( $\mathrm{pH}$-insensitive) transferrin for $10 \mathrm{~min}$ to label endosomes, followed by live imaging (Fig. 4A). A calibration curve was generated as well by imaging of hippocampal neurons preloaded with the fluorescent conjugates of transferrin and then incubated with buffers of known $\mathrm{pH}$ at the time of imaging (Fig. 4B). With respect to endosomes located in the processes of neurons, the $\mathrm{pH}$ of early endosomes in wildtype neurons was $6.48 \pm 0.04$, and in NHE6A11S-mutant neurons was $6.45 \pm 0.06$ ( $n=4$ litters, 9 pups, 4310 cells, 
A

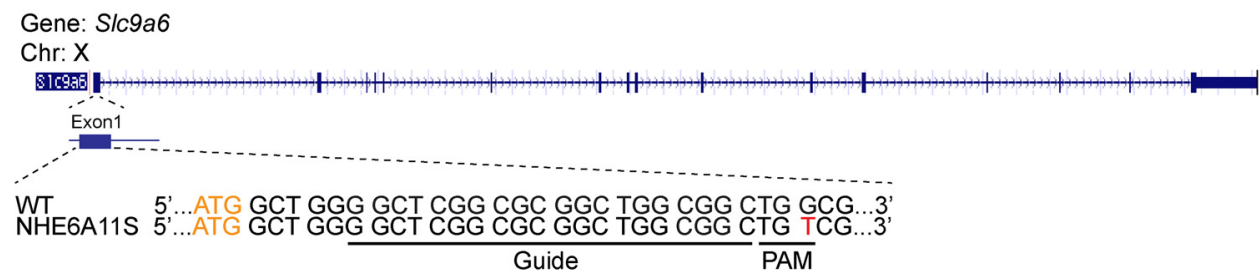

B
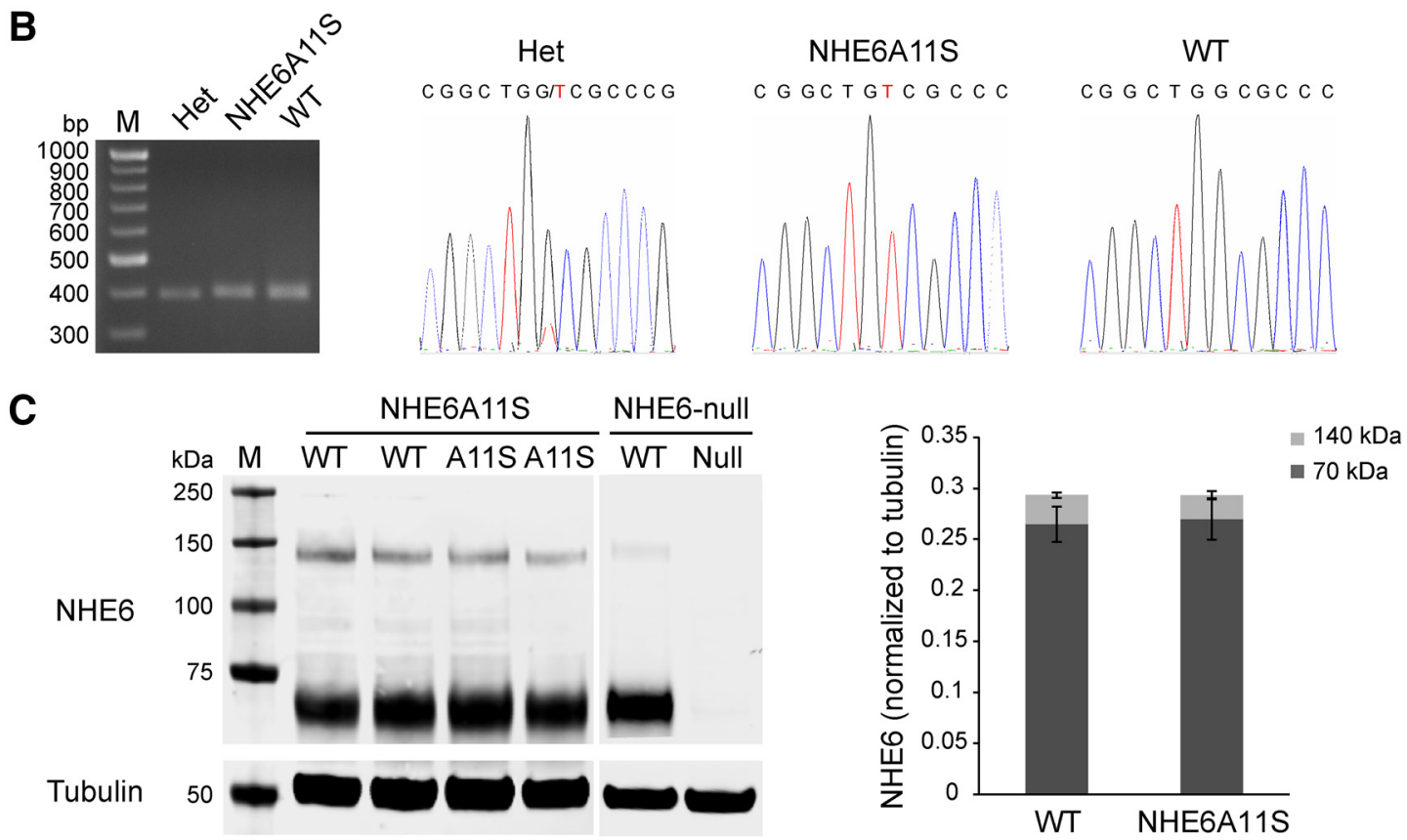

Figure 2. Validation and initial characterization of the NHE6A11S mouse line. $\boldsymbol{A}$, Diagrammatic representation of the targeted exon of mouse SIc9a6 (Nhe6). The target sequence for CRISPR/Cas9-based genome editing is also shown [wild-type (WT), top; NHE6A11S, bottom]. The A11S point mutation $(G>T)$ is in red font; the 20 nt sgRNA target sequence (Guide) and the protospacer adjacent motif (PAM) are underlined; and the start codon is in orange font. $\boldsymbol{B}$, Left, PCR genotyping gel showing a band of 402 bp present in littermate mice of genotypes heterozygous (het), NHE6A11S mutant, and WT. Right, Sanger sequencing chromatograms of the purified $402 \mathrm{bp}$ PCR products confirming the presence of the $\mathrm{G}>\mathrm{T}$ point mutation in samples from the heterozygous and NHE6A11S-mutant mouse lines. The mutant T nucleotide is in red font. $\boldsymbol{C}$, Left, Western blot of mouse brain lysates from WT and mutant mice of the newly generated NHE6A11S and Nhe6-null mouse lines. NHE6 is detected in samples from both WT and mutant mice for the NHE6A11S mouse line, whereas NHE6 is detectable only in the sample from WT mice for the Nhe6-null mouse line. Right, Quantification of Western blots. Quantifications were performed separately for bands at $70 \mathrm{kDa}$ (dark gray) and $140 \mathrm{kDa}$ (light gray). NHE6 expression was normalized to tubulin. Data are presented as the mean \pm SEM. Statistical analyses were conducted using two-tailed Student's $t$ tests. No statistically significant difference in NHE6 levels was detected for samples from WT versus NHE6A11S-mutant mice.

455,578 endosomes for wild-type; $n=4$ litters, 8 pups, 4525 cells, 683,592 endosomes for NHE6A11S; $p=0.67$ ). With respect to endosomes localized in the soma, the $\mathrm{pH}$ of endosomes in wild-type neurons was 6.21 \pm 0.05 , while the $\mathrm{pH}$ of endosomes in NHE6A11S-mutant neurons was $6.14 \pm 0.06$ ( $n=4$ litters, 9 pups, 4310 cells, 47,809 endosomes for wild-type; $n=4$ litters, 8 pups, 4525 cells, $47,276$ endosomes for NHE6A11S; $p=0.38$; Fig. $4 C)$. The endosome area was also analyzed for these same populations of endosomes, with the following results: neurite endosome area: $1.034 \pm 0.01 \mu \mathrm{m}^{2}$ (wild-type); $1.025 \pm$ $0.009 \mu \mathrm{m}^{2}$ (NHE6A11S); soma endosome area: $0.925 \pm$ $0.01 \mu \mathrm{m}^{2}$ (wild-type), $0.914 \pm 0.02 \mu \mathrm{m}^{2}$ (NHE6A11S); $p=$ 0.51 for neurite endosomes, $p=0.57$ for soma endosomes (Fig. 4D). These results indicate that the expression of NHE6A11S does not significantly change the intraendosomal $\mathrm{pH}$ or area of transferrin-labeled endosomes in hippocampal neurons.

\section{Brain development and aging in the NHE6A11S mouse model}

NHE6 has been proposed to play roles in axonal and dendritic branching and in the formation of appropriate brain circuitry. Thus, whether the expression of NHE6A11S might affect related parameters, such as neurite extension and formation of neuronal branch points, was investigated (Fig. 5). For these studies, time-lapse microscopy was performed on cultured hippocampal neurons isolated from P1 pups of wild-type and NHE6A11S-mutant mice using the IncuCyte Live-Cell Imaging System. Using this automated imaging platform, real-time images were generated throughout the culturing 
A Confocal microscopy
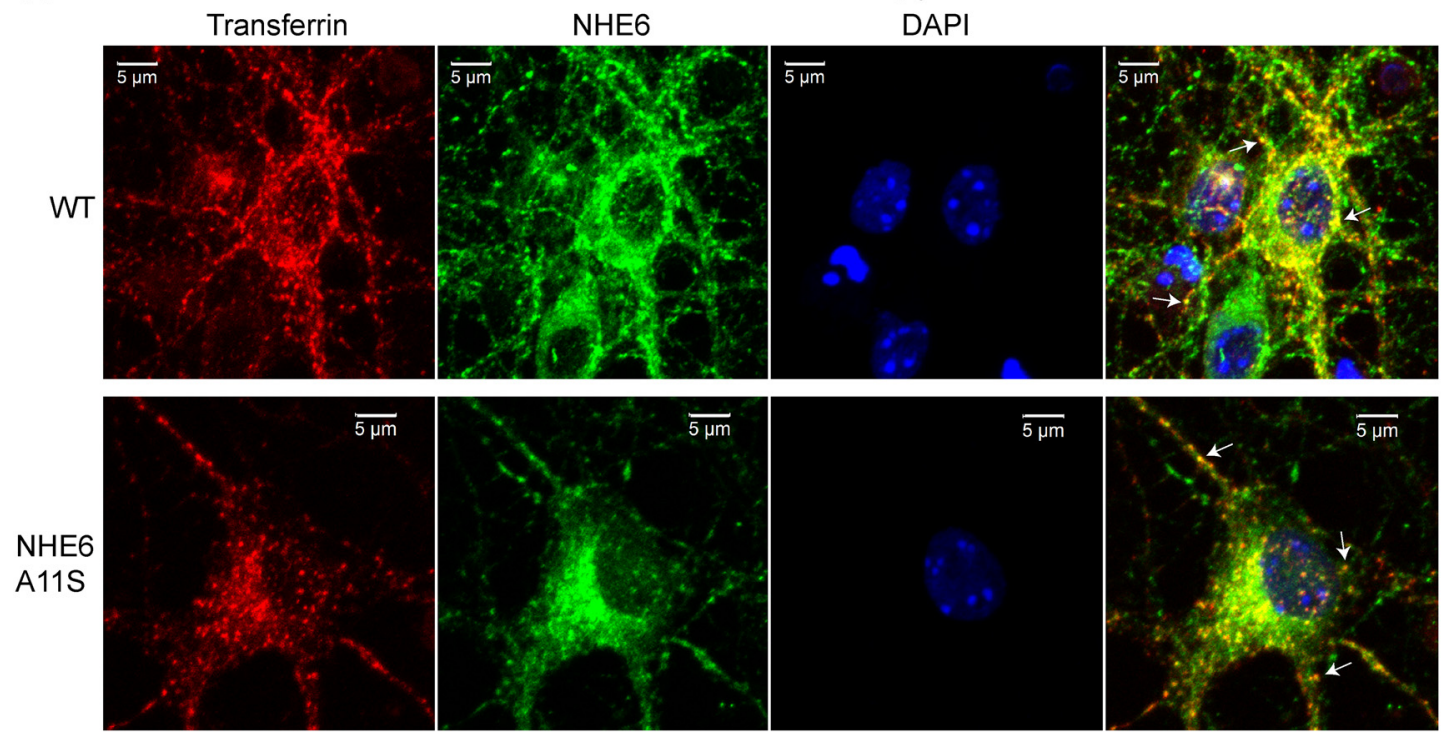

B

Structured illumination microscopy

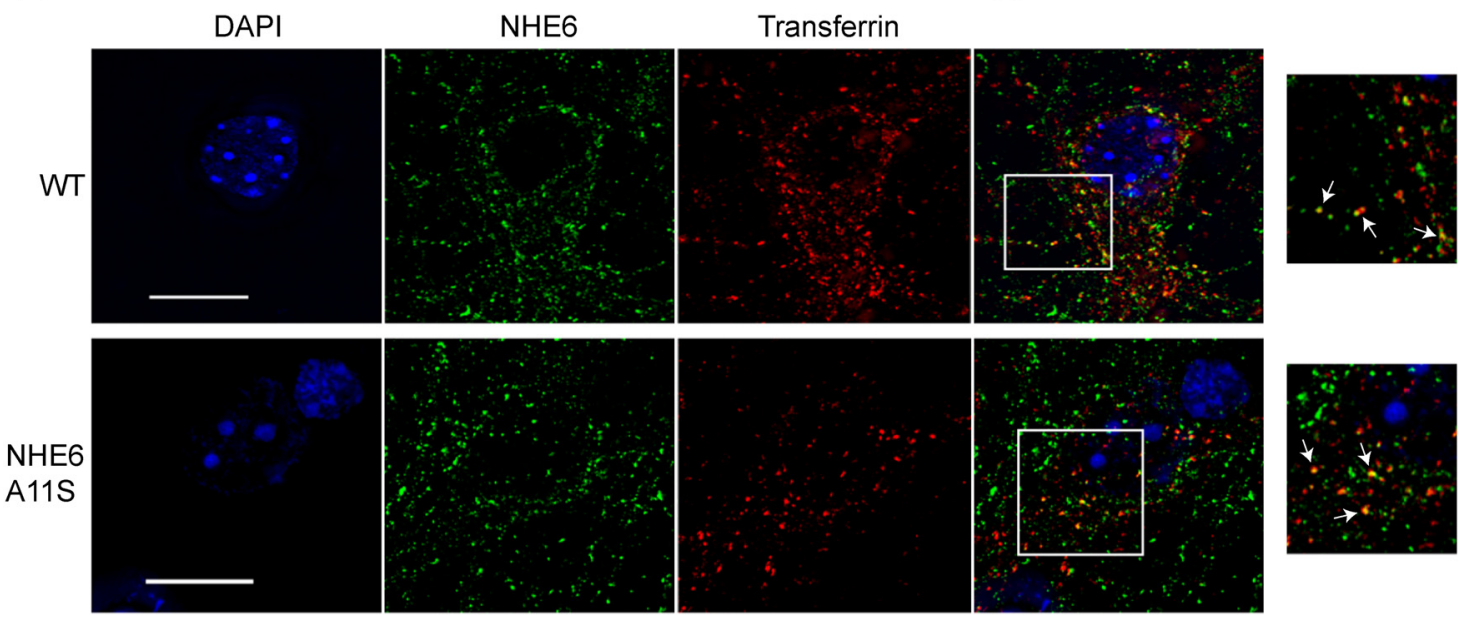

Figure 3. Distribution of NHE6A11S in mouse hippocampal neurons. $\boldsymbol{A}, \boldsymbol{B}$, Fluorescence microscopy images of dissociated hippocampal neurons from wild-type (WT) and NHE6A11S-mutant mice incubated with Alexa Fluor 568-conjugated transferrin (red) for $10 \mathrm{~min}$ to label early endosomes and immunostained for NHE6 (green). Nuclei were counterstained using DAPI (blue). Wide-field confocal microscopy images show colocalization (yellow) of NHE6 and transferrin at presumable early endosomes (arrows; $\boldsymbol{A}$ ). SIM images show areas of colocalization between NHE6 and transferrin (arrows), as well as areas of distinct, nonoverlapping puncta. The areas boxed in white are magnified to the right $(\boldsymbol{B})$. Scale bars, $5 \mu \mathrm{m}$.

of neurons for up to $\sim 15$ DIV (Fig. 5C). Hippocampal cultures isolated from pups of the Nhe6-null mouse line were used as a control as well as for comparison with results obtained from the NHE6A11S mouse line. To this end, consistent with the previous finding of a neuronal arborization defect in Nhe6-null mice (Ouyang et al., 2013), the number of neuronal branch points was significantly reduced in Nhe6-null neurons compared with wildtype neurons (Fig. 5A). Similar defects in neuronal branch points were not, however, observed for NHE6A11Smutant neurons (Fig. 5B), suggesting that the A11S mutation of NHE6 does not affect neurite branching. Neither mutant mouse line showed a defect in neurite length compared with the respective wild-type control (Fig. $5 A, B)$.
In addition to neurodevelopmental defects caused by LoF mutations in SLC9A6, results suggesting neurodegenerative pathology, with age, in CS have been reported (Garbern et al., 2009, 2010; Strømme et al., 2011; Xu et al., 2017). In the mouse, Nhe6-null mice were found to have reductions in brain volume starting at 2 months of age, particularly in the cerebellum. Also, a progressive loss of PCs was observed in Nhe6-null mice, largely starting after 2 months of age and worsening over the course of a year (Strømme et al., 2011; Xu et al., 2017). Given these results reported for the Nhe6-null mouse line, the potential for a neurodegenerative phenotype in the NHE6A11S mouse line was analyzed. In performing direct measurement of gross brain size, no significant difference was detected between brains from wild-type mice and 
A

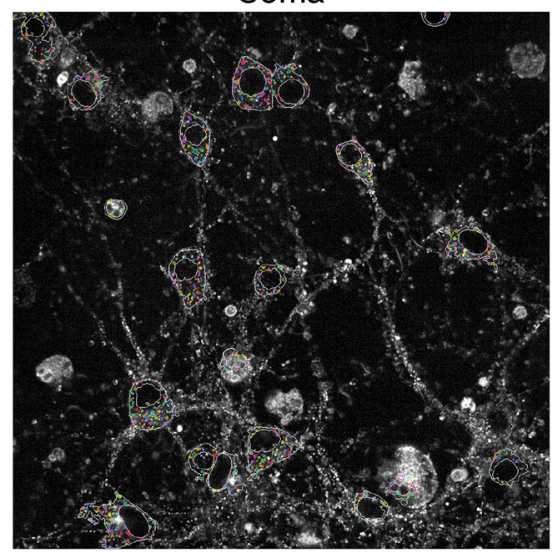

B

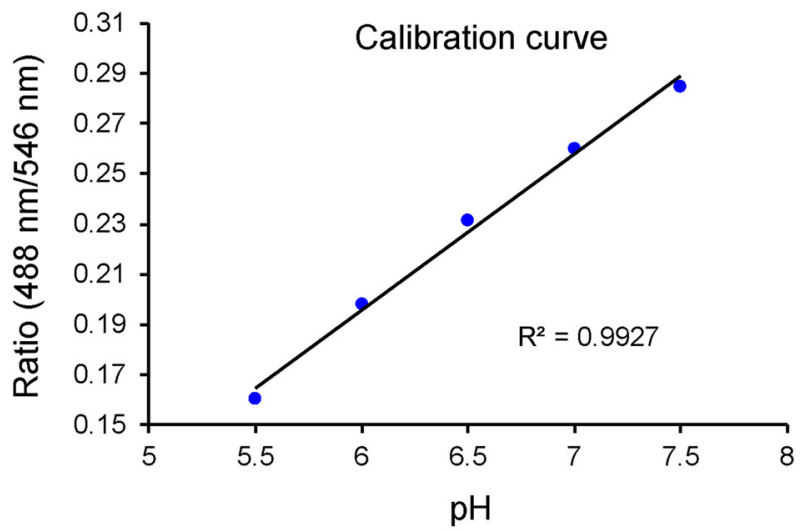

D

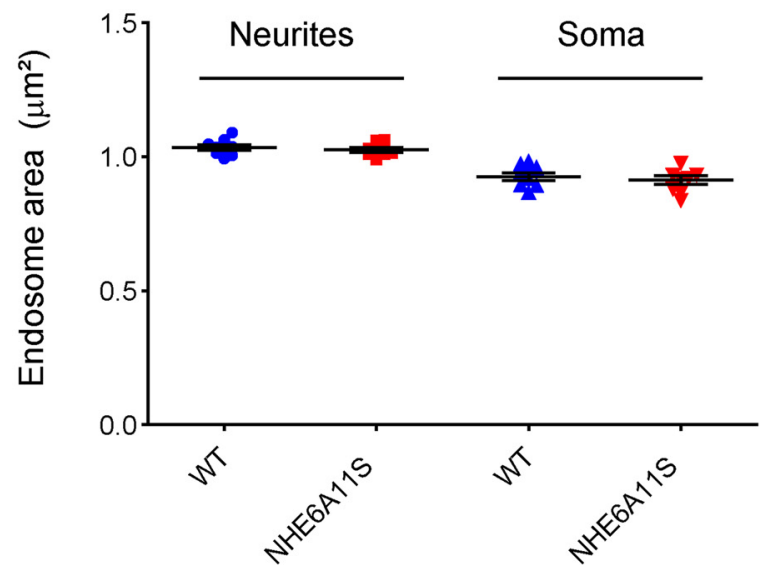

Neurites

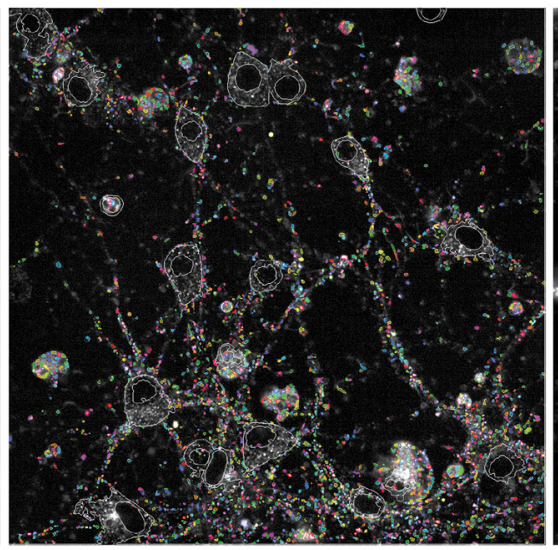

C
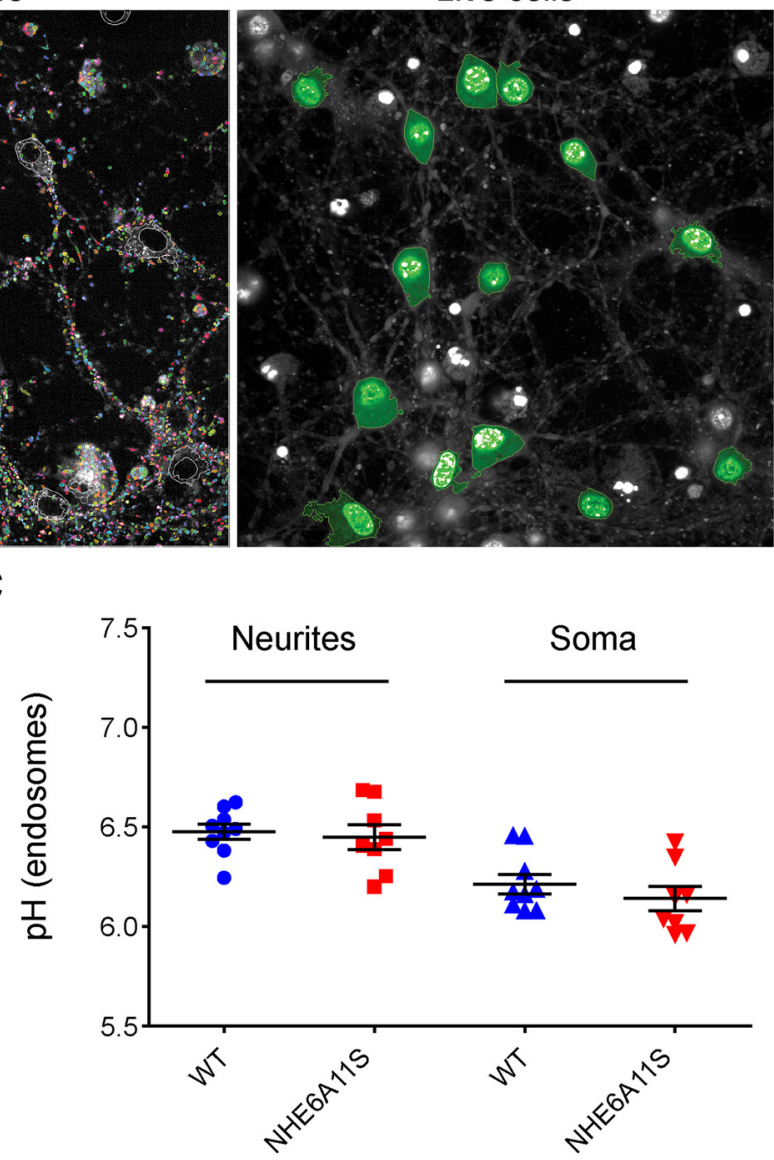

Figure 4. High-throughput confocal microscopy-based evaluation of the effects of NHE6A11S expression on endosomal pH and endosome area in mouse hippocampal neurons. A, Opera Phenix High-Content Screening System images based on a single image of hippocampal neurons showing the fluorescence image and masking for identifying transferrin-labeled endosomes (rainbow color) in soma and neurites. Soma of the identified live cells are demarcated in green (right). Wild-type (WT) and NHE6A11S-mutant hippocampal neurons were loaded simultaneously with FITC-conjugated transferrin and Alexa Fluor 546-conjugated transferrin for 10 min and then imaged under live conditions in regular Neurobasal-A media, $\mathrm{pH}$ 7.4. $\boldsymbol{B}, \mathrm{A} \mathrm{pH}$ calibration curve generated based on images such as those shown in $\boldsymbol{A}$. The wells for generating the $\mathrm{pH}$ calibration curve were incubated with buffers of the indicated $\mathrm{pH}$ values at the time of imaging. $\mathbf{C}$, Luminal $\mathrm{pH}$ values of transferrin-positive endosomes in WT and NHE6A11S-mutant hippocampal neurons determined using the calibration curve shown in $\boldsymbol{B}$. $\boldsymbol{D}$, Areas of transferrin-positive endosomes in WT and NHE6A11S-mutant hippocampal neurons. $\mathrm{pH}$ values and endosome areas were determined for two separate populations of endosomes, those localizing to neurites and those localizing to soma, as indicated. Data are presented as the mean \pm SEM. Statistical analyses were conducted using two-tailed Student's $t$ tests. 
A

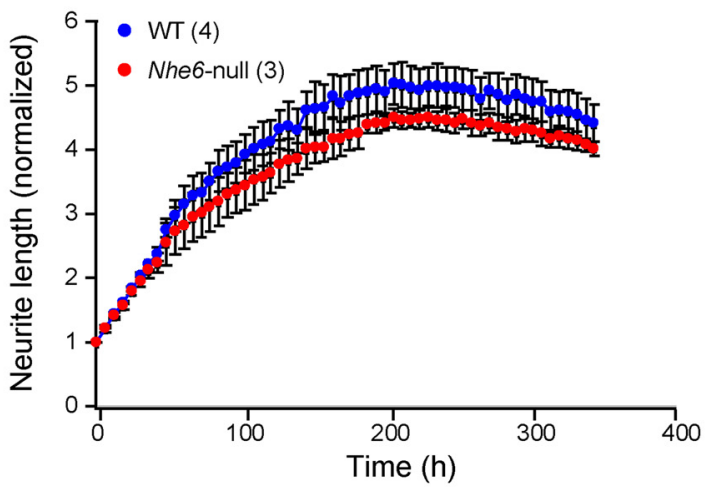

Nhe6-null line

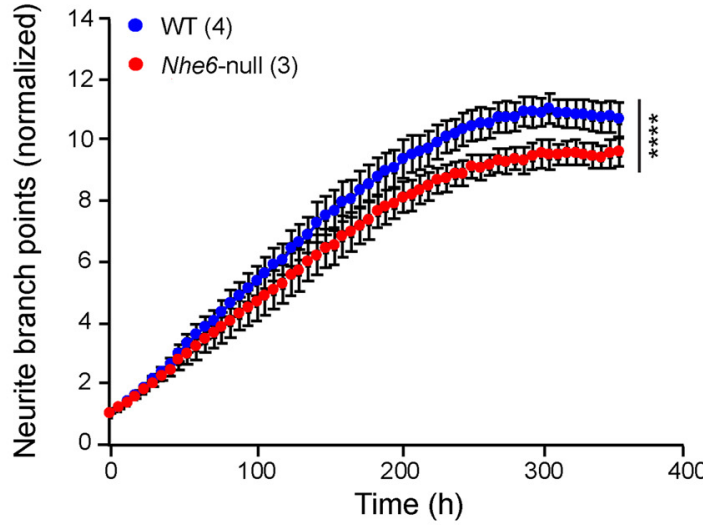

B

NHE6A11S line
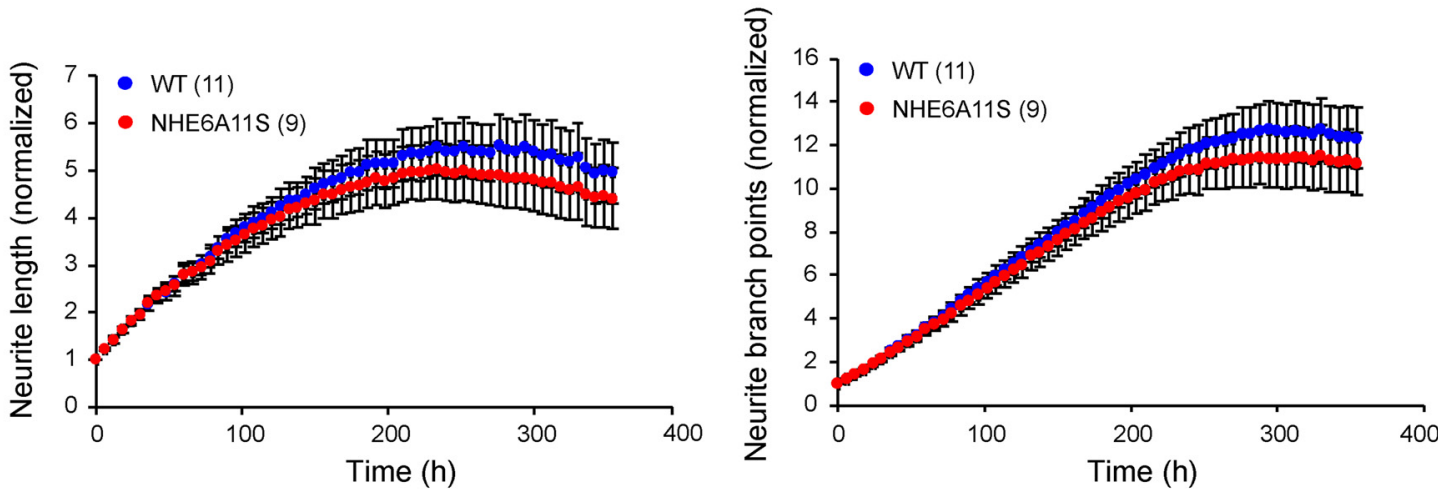

C

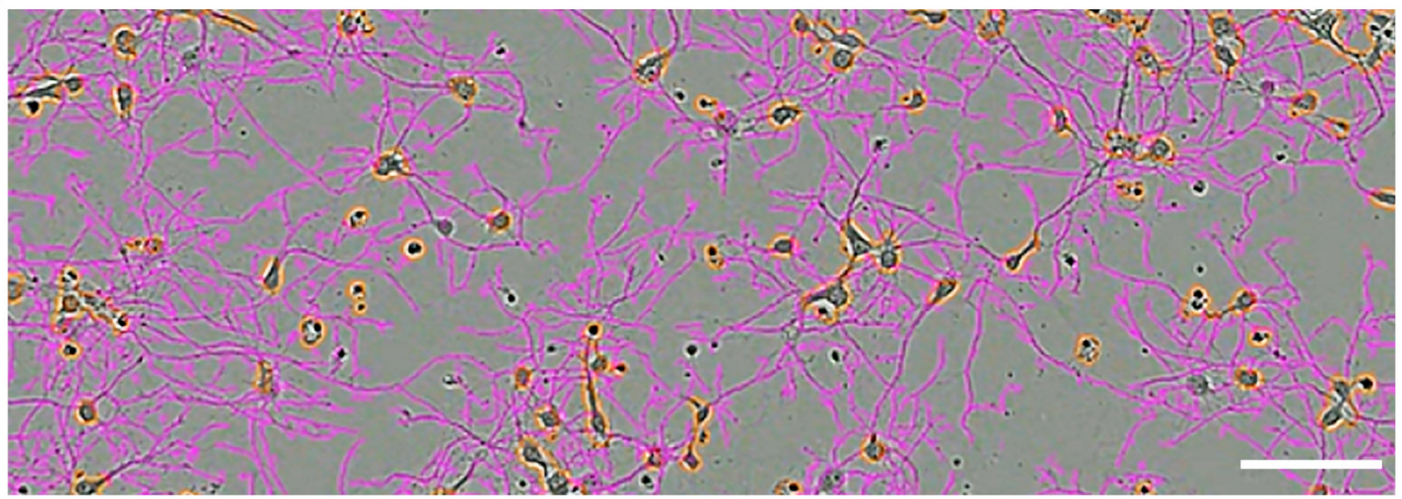

Figure 5. Live imaging-based evaluation of the effects of NHE6A11S expression on neuronal outgrowth. $\boldsymbol{A}, \boldsymbol{B}$, Measurement of neurite outgrowth with respect to neurite length (left) and branch points (right) in hippocampal neurons from an Nhe6-null mouse line $(\boldsymbol{A})$ and the NHE6A11S mouse line (B). Wild-type (WT) and mutant neurons were analyzed for each line. Phase images were acquired from 1 DIV until $\sim 15$ DIV. Neurite length and neurite branch points were normalized to values for these parameters at time 0 h; data are presented as fold $x$ values at time $0 \mathrm{~h}$ (time $0 \mathrm{~h}=1$ ). Data are presented as the mean \pm SEM. Statistical analyses were conducted using two-way ANOVA followed by Tukey's multiple-comparison tests. $* * * * p<0.0001$. C, IncuCyte S3 Live-Cell Analysis System image from a single well of hippocampal neurons showing the phase image and masking for identifying neurites (purple) and cell bodies (orange). Scale bar, $100 \mu \mathrm{m}$.

NHE6A11S-mutant mice at 6 months of age for all examined brain regions (cerebrum, cerebellum, cerebellum + midbrain, and whole brain; Fig. 6A,B). Degeneration of PCs also was not observed in analyzing cerebella from NHE6A11S-mutant mice at 6 months of age that had been immunostained for calbindin, a specific marker for PCs (Fig. $6 C, D)$. These results suggest that brain of the
NHE6A11S-mutant mouse does not undergo neuronal degeneration.

\section{Discussion}

In the present study, a detailed examination of the molecular, cellular, and tissue consequences of a previously identified NHE6 missense variant, potentially causal of AS-like 


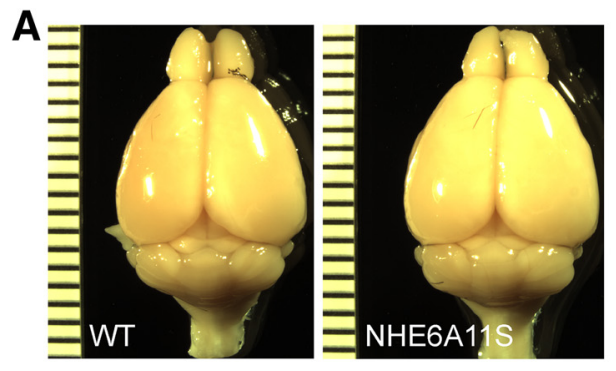

C

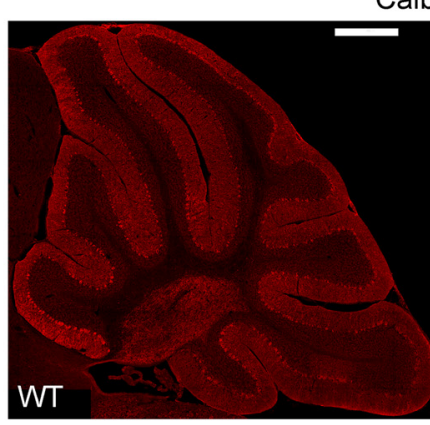

Calbindin

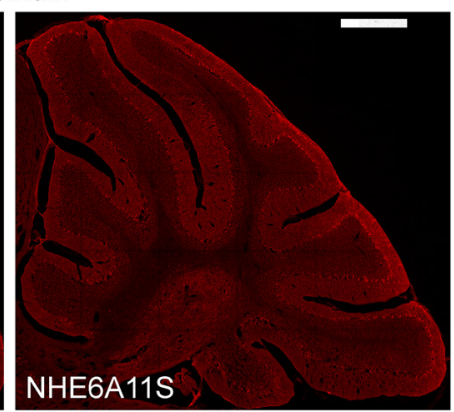

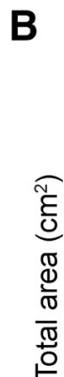

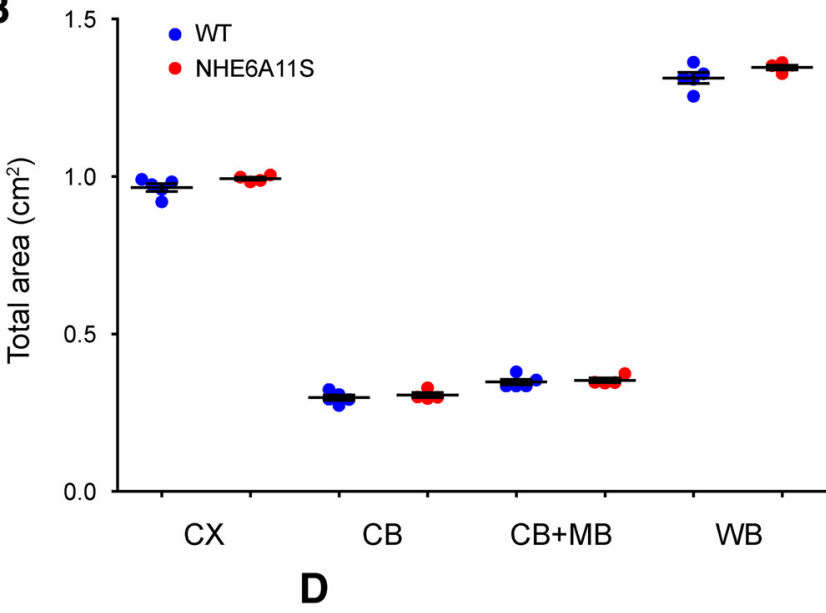

Figure 6. In vivo evaluation of the effects of NHE6A11S expression on mouse brain size and degeneration with aging. $\boldsymbol{A}$, Images of brains from wild-type (WT) and NHE6A11S-mutant mice at 6 months of age. $\boldsymbol{B}$, Quantitative analysis of brain size based on brains from mice at 6 months of age. CX, Cerebrum; CB, cerebellum; CB+MB, cerebellum + midbrain; WB, whole brain. $\boldsymbol{C}$, Confocal microscopy images of $30 \mu \mathrm{m}$ sagittal sections of cerebella from WT and NHE6A11S-mutant mice at 6 months of age immunostained for calbindin, a marker specific to PCs. Scale bars, $500 \mu \mathrm{m}$. $\boldsymbol{D}$, Quantitative analysis of PC density based on cerebellar sections from mice at 6 months of age. Data are presented as the mean \pm SEM. Statistical analyses were conducted using two-tailed Student's $t$ tests. No statistically significant differences in the sizes of the indicated brain regions $(\boldsymbol{B})$ or PC density $(\boldsymbol{D})$ were detected for samples from WT versus NHE6A11S-mutant mice.

features in a male proband, was conducted. The missense mutation-NHE6A9S in human and NHE6A11S in mousesubstitutes a polar serine residue for a highly conserved, nonpolar alanine residue in the $\mathrm{N}$ terminus of NHE6. To perform this overall study, both exogenous expression (in cultured cells) and endogenous expression (in a mouse model) of mutant NHE6 were used and the effects analyzed. Compared with wild-type NHE6, exogenously expressed human NHE6A9S and endogenously expressed mouse NHE6A11S appeared to undergo normal protein synthesis, post-translational maturation, and dimerization. Mutant NHE6 protein also displayed a punctate localization pattern that overlapped with wild-type NHE6 and an endosomal marker, indicating that the alanine-to-serine mutation of NHE6 did not affect its ability to properly localize within cells. The expression of NHE6A11S also did not alter the distribution of transferrin-positive endosomes to neuronal soma versus neurites or appear to affect endosome area. Furthermore, unlike previous reports for Nhe6-null mice (Strømme et al., 2011; Ouyang et al., 2013; Xu et al., 2017), NHE6A11S-mutant mice did not show statistically significant defects in neurite branching or outgrowth, intraendosomal $\mathrm{pH}$, brain size, or PC density. Together, the findings presented here indicate that the alanine-to-serine mutation
(NHE6A9S or NHE6A11S) is not deleterious to the function of NHE6 with respect to these phenotypes.

As whole-genome sequencing and whole-exome sequencing approaches develop, more and more rare mutations are identified. To establish the functional consequences of variants identified in patients, experimental investigation of protein function may be helpful. Note, however, that the designation of a variant as pathogenic is a statistical argument based in human and population genetics (i.e., the presence of variant in affected individuals and the absence of variant in controls). The NHE6A9S variant was reported in a male with an AS-like phenotype but lacking an identified mutation in the relevant gene-UBE3A. The individual is reported as having many of the core symptoms of CS such as profound mental retardation, secondary microcephaly, hyperkinetic behavior, and absent speech. The possibility exists that a variant such as NHE6A9S may confer susceptibility to disease in a complex fashion. For example, a particular variant may not be necessary or sufficient on its own to cause disease but rather it may increase the statistical likelihood of symptom presentation through a model involving interactions with other genetic loci and/or the presence of specific environmental factors. This genetic model has yet to be tested in large case-control or family- 
based studies (i.e., transmission disequilibrium tests). Nonetheless, by screening a large control population in the ExAC database, a greater number of males hemizygous for the NHE6A9S variant were observed than expected, suggesting a lack of selective pressure against this variant in males. Thus, this analysis of population genetic data indicates that the NHE6A9S variant is not pathogenic in a highly penetrant, Mendelian fashion. This does not preclude the possibility that the A9S variant has a functional impact on NHE6, yet with incomplete penetrance; however, the failure to find a fewer number of observed males compared with expected males hemizygous for the NHE6A9S variant argues against this possibility. Combined, the data are suggestive of the concept that the NHE6A9S variant is not involved in disease phenotypes indicative of CS.

All assays for which results are reported here indicate that the NHE6A11S variant does not affect NHE6 function. LoF mutation of NHE6 causes aberrant overacidification of early endosomes. To measure intraendosomal $\mathrm{pH}$, methods involving $\mathrm{pH}$-sensitive fluorophore-conjugated transferrin are frequently used. We developed a highthroughput analysis method using the Opera Phenix HighContent Screening System. Using this method, we found that the A11S mutation of NHE6 did not affect intraendosomal $\mathrm{pH}$. In a prior study, $\sim 83 \%$ of transferrin-positive early endosomes were found to costain with NHE6 (Ouyang et al., 2013). The formal possibility exists that NHE6 mutations could affect an endosome population not captured by this transferrin-based method of determining endosomal pH. However, the Opera Phenix High-Content Screening System allowed us to generate a large dataset through extremely sensitive confocal imaging and collect far more data points than could have been collected in the same amount of time by other reported methods, thereby documenting, with rigor, the absence of a phenotype in the transferrin-positive compartment.

In summary, the results reported here based in population genetics indicate that the NHE6A9S variant is unlikely to be pathogenic in a highly penetrant, Mendelian fashion. Results from mouse studies using a newly generated NHE6A11S mouse line are also supportive of this conclusion with respect to the phenotypes analyzedneuronal arborization, intraendosomal $\mathrm{pH}$, brain size, and PC density. More generally, the study presented here demonstrates a novel research pipeline for the evaluation of NHE6 variants found in CS patients, including the evaluation of the functional effects of variants in vivo when expressed under the endogenous promoter.

\section{References}

Allen Institute for Brain Science (2004) Allen mouse brain atlas. Seattle, WA: Allen Institute for Brain Science.

Bosemani T, Zanni G, Hartman AL, Cohen R, Huisman TA, Bertini E, Poretti A (2014) Christianson syndrome: spectrum of neuroimaging findings. Neuropediatrics 45:247-251.

Brett CL, Wei Y, Donowitz M, Rao R (2002) Human $\mathrm{Na}(+) / \mathrm{H}(+)$ exchanger isoform 6 is found in recycling endosomes of cells, not in mitochondria. Am J Physiol Cell Physiol 282:C1031-C1041.

Christianson AL, Stevenson RE, van der Meyden $\mathrm{CH}$, Pelser J, Theron FW, van Rensburg PL, Chandler M, Schwartz CE (1999) X linked severe mental retardation, craniofacial dysmorphology, epilepsy, ophthalmoplegia, and cerebellar atrophy in a large South African kindred is localised to Xq24-q27. J Med Genet 36:759766.

Deane EC, Ilie AE, Sizdahkhani S, Das Gupta M, Orlowski J, McKinney RA (2013) Enhanced recruitment of endosomal $\mathrm{Na}^{+} / \mathrm{H}^{+}$ exchanger NHE6 into dendritic spines of hippocampal pyramidal neurons during NMDA receptor-dependent long-term potentiation. J Neurosci 33:595-610.

Fichou Y, Bahi-Buisson N, Nectoux J, Chelly J, Héron D, Cuisset L, Bienvenu T (2009) Mutation in the SLC9A6 gene is not a frequent cause of sporadic Angelman-like syndrome. Eur J Hum Genet $17: 1378-1380$

Garbern J, Neumann M, Trojanowski JQ, Lee VMY, Schwartz CE, Feldman GE, Kupsky WAF, Sima AAF (2009) X-linked corticobasal degeneration and tau deposition in a family with severe autism and mutation in the SLC9A6 gene. Neurology 72:A256.

Garbern JY, Neumann M, Trojanowski JQ, Lee VM, Feldman G, Norris JW, Friez MJ, Schwartz CE, Stevenson R, Sima AA (2010) A mutation affecting the sodium/proton exchanger, SLC9A6, causes mental retardation with tau deposition. Brain 133:1391-1402.

Gilfillan GD, Selmer KK, Roxrud I, Smith R, Kyllerman M, Eiklid K, Kroken $M$, Mattingsdal M, Egeland T, Stenmark $H$, Sjøholm $H$, Server A, Samuelsson L, Christianson A, Tarpey P, Whibley A, Stratton MR, Futreal PA, Teague J, Edkins S, et al (2008) SLC9A6 mutations cause $\mathrm{X}$-linked mental retardation, microcephaly, epilepsy, and ataxia, a phenotype mimicking Angelman syndrome. Am J Hum Genet 82:1003-1010.

Ilie A, Weinstein E, Boucher A, McKinney RA, Orlowski J (2014) Impaired posttranslational processing and trafficking of an endosomal $\mathrm{Na}+/ \mathrm{H}+$ exchanger NHE6 mutant $(\Delta(370) \mathrm{WST}(372))$ associated with $\mathrm{X}$-linked intellectual disability and autism. Neurochem Int 73:192-203.

Ilie A, Gao AY, Reid J, Boucher A, McEwan C, Barrière H, Lukacs GL, McKinney RA, Orlowski J (2016) A Christianson syndrome-linked deletion mutation $(\Delta(287) \mathrm{ES}(288))$ in SLC9A6 disrupts recycling endosomal function and elicits neurodegeneration and cell death. Mol Neurodegener 11:63.

Ilie A, Gao AYL, Boucher A, Park J, Berghuis AM, Hoffer MJV, Hilhorst-Hofstee Y, McKinney RA, Orlowski J (2019) A potential gain-of-function variant of SLC9A6 leads to endosomal alkalinization and neuronal atrophy associated with Christianson syndrome. Neurobiol Dis 121:187-204.

Kerner-Rossi M, Gulinello M, Walkley S, Dobrenis K (2018) Pathobiology of Christianson syndrome: linking disrupted endosomallysosomal function with intellectual disability and sensory impairments. Neurobiol Learn Mem 14:106867.

Kondapalli KC, Prasad H, Rao R (2014) An inside job: how endosomal $\mathrm{Na}(+) / \mathrm{H}(+)$ exchangers link to autism and neurological disease. Front Cell Neurosci 8:172.

Lek M, Karczewski KJ, Minikel EV, Samocha KE, Banks E, Fennell T, O'Donnell-Luria AH, Ware JS, Hill AJ, Cummings BB, Tukiainen T, Birnbaum DP, Kosmicki JA, Duncan LE, Estrada K, Zhao F, Zou J, Pierce-Hoffman E, Berghout J, Cooper DN, et al (2016) Analysis of protein-coding genetic variation in 60,706 humans. Nature 536 : 285-291.

Masurel-Paulet A, Piton A, Chancenotte S, Redin C, Thauvin-Robinet $C$, Henrenger $Y$, Minot D, Creppy A, Ruffier-Bourdet M, Thevenon J, Kuentz P, Lehalle D, Curie A, Blanchard G, Ghosn E, Bonnet M, Archimbaud-Devilliers M, Huet F, Perret O, Philip N, Mandel JL, et al (2016) A new family with an SLC9A6 mutation expanding the phenotypic spectrum of Christianson syndrome. Am J Med Genet A 170:2103-2110.

Mignot C, Héron D, Bursztyn J, Momtchilova M, Mayer M, Whalen S, Legall A, Billette de Villemeur T, Burglen $L$ (2013) Novel mutation in SLC9A6 gene in a patient with Christianson syndrome and retinitis pigmentosum. Brain Dev 35:172-176.

Nakamura N, Tanaka S, Teko Y, Mitsui K, Kanazawa H (2005) Four $\mathrm{Na}+/ \mathrm{H}+$ exchanger isoforms are distributed to Golgi and post- 
Golgi compartments and are involved in organelle $\mathrm{pH}$ regulation. J Biol Chem 280:1561-1572.

National Research Council of the National Academies (2011) Guide for the care and use of laboratory animals, Ed 8. Washington, DC: The National Academies.

Ohgaki R, Fukura N, Matsushita M, Mitsui K, Kanazawa H (2008) Cell surface levels of organellar $\mathrm{Na}+/ \mathrm{H}+$ exchanger isoform 6 are regulated by interaction with RACK1. J Biol Chem 283:4417-4429.

Ohgaki R, Matsushita M, Kanazawa H, Ogihara S, Hoekstra D, van ljzendoorn SC (2010) The $\mathrm{Na}+/ \mathrm{H}+$ exchanger NHE6 in the endosomal recycling system is involved in the development of apical bile canalicular surface domains in HepG2 cells. Mol Biol Cell 21:1293-1304.

Ohgaki R, van ljzendoorn SC, Matsushita M, Hoekstra D, Kanazawa $\mathrm{H}$ (2011) Organellar $\mathrm{Na}+/ \mathrm{H}+$ exchangers: novel players in organelle $\mathrm{pH}$ regulation and their emerging functions. Biochemistry 50:443-450.

Orlowski J, Grinstein S (2004) Diversity of the mammalian sodium/ proton exchanger SLC9 gene family. Pflugers Arch 447:549-565.

Ouyang Q, Lizarraga SB, Schmidt M, Yang U, Gong J, Ellisor D, Kauer JA, Morrow EM (2013) Christianson syndrome protein NHE6 modulates TrkB endosomal signaling required for neuronal circuit development. Neuron 80:97-112.

Padmanabha H, Saini AG, Sahu JK, Singhi P (2017) Syndrome of X linked intellectual disability, epilepsy, progressive brain atrophy and large head associated with SLC9A6 mutation. BMJ Case Rep 2017:bcr-2017-222050.

Pescosolido MF, Stein DM, Schmidt M, El Achkar CM, Sabbagh M, Rogg JM, Tantravahi U, McLean RL, Liu JS, Poduri A, Morrow EM (2014) Genetic and phenotypic diversity of NHE6 mutations in Christianson syndrome. Ann Neurol 76:581-593.

Riess A, Rossier E, Krüger R, Dufke A, Beck-Woedl S, Horber V, Alber M, Gläser D, Riess O, Tzschach A (2013) Novel SLC9A6 mutations in two families with Christianson syndrome. Clin Genet 83:596-597.

Roxrud I, Raiborg C, Gilfillan GD, Strømme P, Stenmark H (2009) Dual degradation mechanisms ensure disposal of NHE6 mutant protein associated with neurological disease. Exp Cell Res 315: 3014-3027.

Schroer RJ, Holden KR, Tarpey PS, Matheus MG, Griesemer DA, Friez MJ, Fan JZ, Simensen RJ, Strømme P, Stevenson RE, Stratton MR, Schwartz CE (2010) Natural history of Christianson syndrome. Am J Med Genet A 152A:2775-2783.

Schuurs-Hoeijmakers JH, Vulto-van Silfhout AT, Vissers LE, van de V II, van Bon BW, de Ligt J, Gilissen C, Hehir-Kwa JY, Neveling K, del Rosario M, Hira G, Reitano S, Vitello A, Failla P, Greco D,
Fichera M, Galesi O, Kleefstra T, Greally MT, Ockeloen CW, et al (2013) Identification of pathogenic gene variants in small families with intellectually disabled siblings by exome sequencing. J Med Genet 50:802-811.

Strømme P, Dobrenis K, Sillitoe RV, Gulinello M, Ali NF, Davidson C, Micsenyi MC, Stephney G, Ellevog L, Klungland A, Walkley SU (2011) X-linked Angelman-like syndrome caused by Slc9a6 knockout in mice exhibits evidence of endosomal-lysosomal dysfunction. Brain 134:3369-3383.

Takahashi Y, Hosoki K, Matsushita M, Funatsuka M, Saito K, Kanazawa H, Goto Y, Saitoh S (2011) A loss-of-function mutation in the SLC9A6 gene causes $X$-linked mental retardation resembling Angelman syndrome. Am J Med Genet B Neuropsychiatr Genet 156B:799-807.

Tarpey PS, Smith R, Pleasance E, Whibley A, Edkins S, Hardy C, O'Meara S, Latimer C, Dicks E, Menzies A, Stephens P, Blow M, Greenman C, Xue Y, Tyler-Smith C, Thompson D, Gray K, Andrews J, Barthorpe S, Buck G, et al (2009) A systematic, largescale resequencing screen of $\mathrm{X}$-chromosome coding exons in mental retardation. Nat Genet 41:535-543.

Trump N, McTague A, Brittain H, Papandreou A, Meyer E, Ngoh A, Palmer R, Morrogh D, Boustred C, Hurst JA, Jenkins L, Kurian MA, Scott $\mathrm{RH}$ (2016) Improving diagnosis and broadening the phenotypes in early-onset seizure and severe developmental delay disorders through gene panel analysis. J Med Genet 53:310-317.

Tzschach A, Ullmann R, Ahmed A, Martin T, Weber G, DeckerSchwering O, Pauly F, Shamdeen MG, Reith W, Oehl-Jaschkowitz B (2011) Christianson syndrome in a patient with an interstitial Xq26.3 deletion. Am J Med Genet A 155A:2771-2774.

Weitensteiner V, Zhang R, Bungenberg J, Marks M, Gehlen J, Ralser DJ, Hilger AC, Sharma A, Schumacher J, Gembruch U, Merz WM, Becker A, Altmüller J, Thiele H, Herrmann BG, Odermatt B, Ludwig $M$, Reutter $\mathrm{H}$ (2018) Exome sequencing in syndromic brain malformations identifies novel mutations in ACTB, and SLC9A6, and suggests BAZ1A as a new candidate gene. Birth Defects Res 110:587-597.

Xu M, Ouyang Q, Gong J, Pescosolido MF, Pruett BS, Mishra S, Schmidt M, Jones RN, Gamsiz Uzun ED, Lizarraga SB, Morrow EM (2017) Mixed neurodevelopmental and neurodegenerative pathology in Nhe6-null mouse model of Christianson syndrome. eNeuro 4:ENEURO.0388-0317.2017.

Zanni G, Barresi S, Cohen R, Specchio N, Basel-Vanagaite L, Valente EM, Shuper A, Vigevano F, Bertini E (2014) A novel mutation in the endosomal $\mathrm{Na}+/ \mathrm{H}+$ exchanger NHE6 (SLC9A6) causes Christianson syndrome with electrical status epilepticus during slow-wave sleep (ESES). Epilepsy Res 108:811-815. 